\title{
Phytochemicals as Dynamic Surface Ligands To Control Nanoparticle-Protein Interactions
}

\author{
Amanda N. Abraham, ${ }^{\dagger}$ Tarun K. Sharma, ${ }^{\dagger}$ Vipul Bansal, $*,{ }^{\dagger}$ and Ravi Shukla ${ }^{*}, \dagger$ \\ ${ }^{\dagger}$ Ian Potter NanoBioSensing Facility, NanoBiotechnology Research Lab (NBRL), School of Science, and ${ }^{\ddagger}$ Centre for Advanced \\ Materials \& Industrial Chemistry, School of Science, RMIT University, GPO Box 2476 V, Melbourne, Victoria 3001, Australia
}

\section{Supporting Information}

ABSTRACT: The rapid formation of the protein corona on to the nanoparticle (NP) surface is the key that confers biological identity to NPs and subsequently dictates their fate both in vitro and in vivo. Despite significant efforts, the inability to control the spontaneous interaction of serum proteins with the administered NPs remains a major constraint in clinical translation of nanomedicines. The ligands present on the NP surface offer promise in controlling their biological interactions; however, their influence on the NP-protein interaction is not well-understood. The current study investigates the potential of phytochemical-capped silver nanoparticles (AgNPs) toward allowing a control over NP interactions with the human serum albumin (HSA), the most abundant protein in the biological fluids. Specifically, we demonstrate the ability of curcumin (Cur) and epigallocatechin-3gallate (EGCG) to independently act as reducing agents to produce phytochemical-capped AgNPs that show biologically desirable interactions with HSA. The key finding of our study is that the phytochemical-capped AgNPs initially interact with HSA more strongly compared to the citrate-stabilized AgNPs; however, the resultant NP-HSA complexes are less stable in the case of the former, which causes a lesser degree of changes in the protein conformation during interactions. Further, the choice of the phytochemical allows control over NP-HSA interactions, such that Cur- and EGCG-capped AgNPs interacted with HSA in a static versus dynamic manner, respectively. The diversity of the functional groups present in natural phytochemicals and their potential as in situ capping ligands during synthesis offer new opportunities in controlling the interactions of NPs with complex biological fluids, with implications in nanodiagnostics and nanomedicine.

\section{INTRODUCTION}

The unique physicochemical and broad spectrum antibacterial and antifungal properties of silver nanoparticles (AgNPs) have made them one of the most incorporated nanomaterials in the consumer and medical products to date. ${ }^{1,2}$ However, on administering these NPs into the blood stream, they first come into contact with proteins found in the plasma. ${ }^{3}$ These plasma proteins readily interact with the surface of the NPs forming a protein corona (PC). ${ }^{4}$ This initial interaction between the NPs and plasma proteins changes the properties of the NPs, and as such, this has remarkable impact on altering the mechanism of NP interaction with the target organs. ${ }^{5}$ Hence, distribution and biological responses of the NPs in the body are dictated by this PC because this altered configuration of NPs is what that is perceived when the NPs first come in contact with the target cells. ${ }^{4,6,7}$ The NPs, in turn, can also change the conformation of the plasma proteins they interact with, possibly altering the function of these proteins. ${ }^{1}$ Hence, when considering the use of NPs for in vivo purposes, it is important to understand and control these interactions of NPs with proteins in the blood stream.
Albumins are the most abundant proteins found in the human serum, at a concentration of $769 \mu \mathrm{M} / \mathrm{L} .{ }^{8}$ Human serum albumin (HSA) contains 585 amino acid residues, and it serves as an important carrier for many substances such as fatty acids, bilirubins, hormones, and exogenous and endogenous ligands. ${ }^{9}$ Because HSA possesses so many important physiological functions, any change to its structure can prove detrimental to its normal functioning in the body. ${ }^{1,10,11}$ Hence, it is important to study the interaction of NPs with this protein. In particular, HSA contains a single tryptophan (Trp-214) residue in the hydrophobic cavity of its sub-domain IIA (Sudlow I). This Trp residue is capable of producing strong intrinsic fluorescence and therefore serves as an excellent reporter for ligand binding and conformational studies. ${ }^{1,12}$

Notably, while there are some studies that have utilized human serum or simulated complex biological environments, ${ }^{13,14}$ most studies that have looked at NP-protein interactions have utilized bovine serum albumin $(\mathrm{BSA})^{3,6,15-18}$

Received: November 28, 2017

Accepted: February 5, 2018

Published: February 22, 2018 

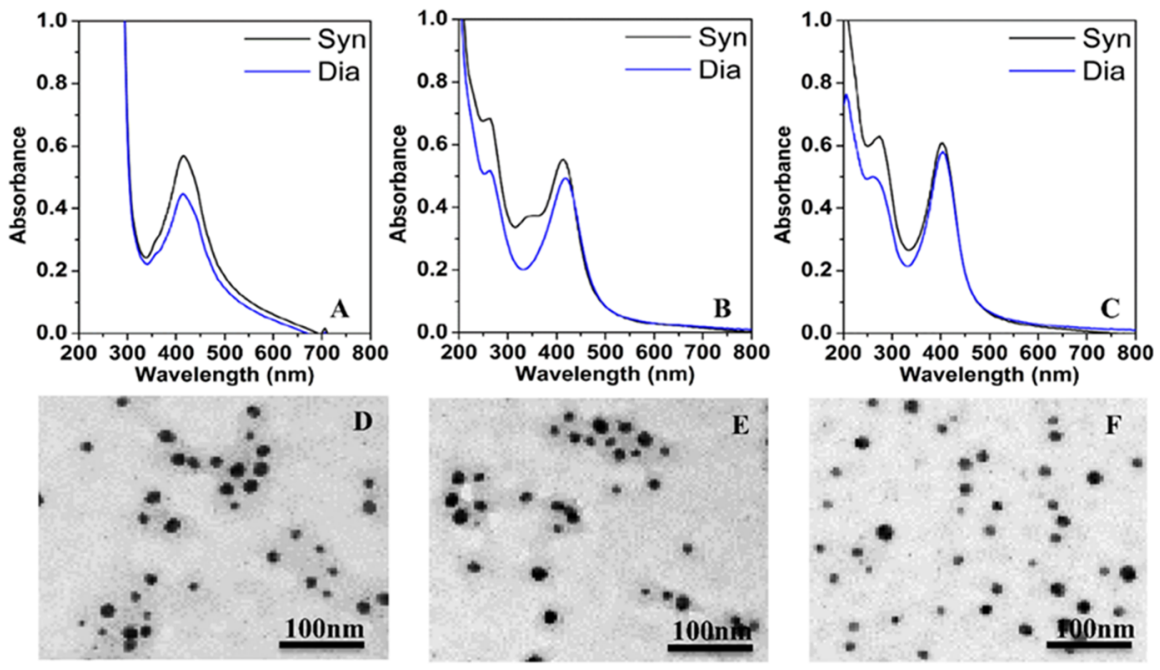

Figure 1. UV-vis absorbance spectra (A-C) of as-synthesized (Syn) and post-dialysis (Dia) nanoparticles and corresponding post-dialysis TEM images (D-F) of Ct-AgNPs (A,D), Cur-AgNPs (B,E), and EGCG-AgNPs (C,F).

instead of HSA. However, one important difference between BSA and HSA is the presence of two tryptophan residues in BSA, whereas HSA has a single unique tryptophan residue. ${ }^{12,19}$ Owing to this inherent difference, these two proteins may show different binding interactions with NPs. For instance, Gelamo and Tabak $^{19}$ noted that in the presence of various ionic surfactants, BSA evinced fluorescence quenching, whereas HSA led to an enhancement of the fluorescence with the same ionic surfactants. Manivel and Anandan ${ }^{2}$ also observed that BSA can exhibit higher binding with NPs than HSA. This supports that BSA is not a reliable substitute for HSA, especially when employing fluorescence for fundamental interaction studies. As such, in contrast to a serum protein of bovine origin, HSA offers a more appropriate platform to study and understand the NP interactions in the human context. Hence, our current study focuses on studying the interaction of HSA with NPs to obtain insights into the likely fate of NPs in the human blood/ serum.

On the other hand, the NP surface characteristics, viz., the biomolecules or phytochemicals present on the surface, may also affect the degree and type of serum protein interactions. Isolated phytochemicals, such as curcumin, ${ }^{20}$ epigallocatechin3-gallate (EGCG), ${ }^{21}$ and various flavonoids, ${ }^{22}$ have been observed to show interactions with serum. However, the study of the interaction of phytochemical-coated NPs remains elusive. It is important to study the direct interaction of phytochemical-coated NPs with serum proteins, as it is not uncommon for the functional groups of the phytochemicals to be modified during the process of NP synthesis and capping. ${ }^{23}$ This altered structure of phytochemical bound onto the NP surface could in turn influence the mode of interactions with serum proteins. It is also a focus of our current study to understand how this change in the phytochemical structure might affect the interaction of these NPs with proteins in the blood. To obtain insights into these fundamental questions, we utilize curcumin and EGCG phytochemicals found in turmeric and green tea, respectively, to synthesize AgNPs and elucidate their physicochemical interactions with HSA. We chose curcumin and EGCG as ligands in the current study, as these two phytochemicals have been actively investigated for their therapeutic potential. For instance, EGCG has been recently exploited as a ligand to selectively target Laminin-67 receptor, a protein overexpressed on the surface of prostate cancer cells. ${ }^{24}$ The EGCG functionalization on the surface of gold NPs led to selective uptake of these NPs into cancer cells which could be employed for therapeutic purpose. Similarly, the use of curcumin-based nanoformulations has been actively proposed for a number of biomedical applications ranging from therapy of cancer to cardiovascular diseases, Alzheimer's disease, inflammations, and neurological disorders. ${ }^{25}$

\section{RESULTS AND DISCUSSION}

Synthesis and Characterization of PhytochemicalCoated AgNPs. Phytochemical-coated AgNPs were synthesized and characterized by UV-vis absorbance spectroscopy, transmission electron microscopy (TEM), dynamic light scattering (DLS), and zeta-potential measurements. Figure $1 \mathrm{~A}-\mathrm{C}$ compares the UV-vis absorbance spectra of the assynthesised AgNPs using different reducing agents and those obtained after dialysis to remove potentially unutilized precursors. AgNPs obtained using citrate, curcumin, and EGCG as reducing agents have been referred as Ct-AgNPs, Cur-AgNPs, and EGCG-AgNPs throughout this study. The assynthesized AgNPs display characteristic surface plasmon resonance (SPR) bands ca. 405-416 nm (408 nm for CtAgNPs, $416 \mathrm{~nm}$ for Cur-AgNPs, and $406 \mathrm{~nm}$ for EGCGAgNPs). ${ }^{3,26}$ Additional absorption features are observed at ca. 260-280 nm only in the case of phytochemical-stabilized NPs. Supporting Information Figure $\mathrm{S} 1$ shows the absorbance features of curcumin at 263 and $417 \mathrm{~nm}^{27}$ and EGCG at 235 and $273 \mathrm{~nm} .{ }^{28}$ Hence, the $263 \mathrm{~nm}$ peak for Cur-AgNPs and the $273 \mathrm{~nm}$ peak for EGCG-AgNPs can be ascribed to the electronic transitions from the benzene ring present in these phytochemicals. Subsequent to the dialysis performed on these NPs, the free phytochemicals are removed, leading to a decrease in the intensity of the absorption peaks corresponding to these phytochemicals. The high intensity absorption peak observed ca. $209 \mathrm{~nm}$ in the case of Ct-AgNPs possibly stems from the citrate on the NPs because trisodium citrate shows an absorption band at $209 \mathrm{~nm}$ (Supporting Information Figure S1). ${ }^{29}$ The resulting absorbance features seen in the UV range of the spectra after dialysis stem from the citrate and phytochemicals firmly bound to the surface of AgNPs. 

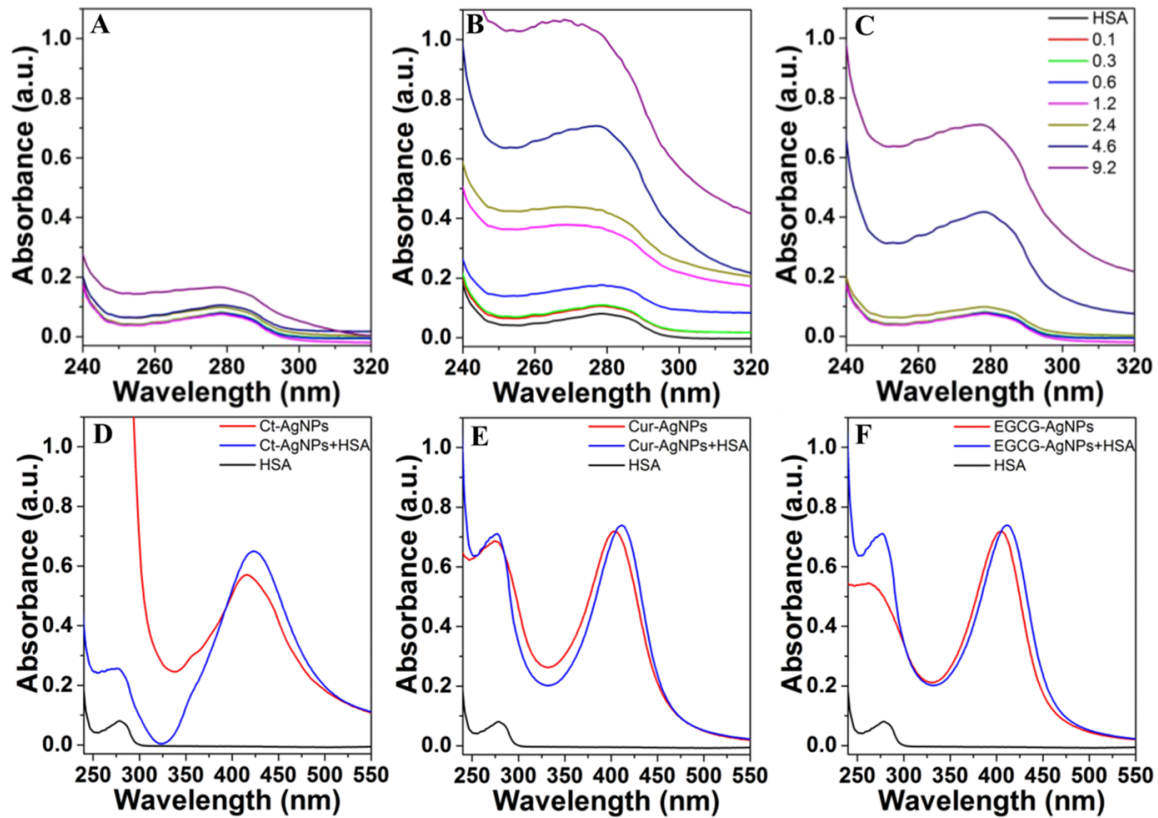

Figure 2. UV-vis absorption spectra in (A-C) depicting interactions of a fixed concentration of HSA $(3 \mu \mathrm{M})$ with an increasing concentration of AgNPs $(0-9.2 \mu \mathrm{M})$ in the HSA absorption region. HSA in the figure corresponds to the equivalent concentration of HSA without nanoparticles. (D-F) depict changes in the SPR absorbance of AgNPs $(1.2 \mu \mathrm{M})$ on exposure to HSA $(3 \mu \mathrm{M})$. (A,D) Ct-AgNPs, (B,E) Cur-AgNPs, and (C,F) EGCG-AgNPs.

The phytochemical-coated AgNPs as well as Ct-AgNPs were of narrow size distribution with a mean diameter of $10-20 \mathrm{~nm}$, as confirmed by TEM (Figure $1 \mathrm{D}-\mathrm{F}$ ). Previous studies have noted that NPs $<50 \mathrm{~nm}$ in diameter are readily internalized into cells, ${ }^{30}$ suggesting that AgNPs used in the current study are in an appropriate size range for potential biological applications. The hydrodynamic diameters for Ct-AgNPs, Cur-AgNPs, and EGCG-AgNPs were recorded to be 55.6, 44.8, and $61.5 \mathrm{~nm}$, respectively; and the corresponding zeta potentials were -25.9 , -41.3, and $-38.9 \mathrm{mV}$ (Supporting Information, Table S1).

As a preliminary method of determining the interaction of HSA with AgNPs, the changes in the DLS and zeta potential of NPs were monitored in the presence and absence of HSA. The hydrodynamic diameters of AgNPs increase in the presence of HSA, accompanied with a concomitant decrease in the zeta potential (Supporting Information, Table S1). The Ct-AgNPs and EGCG-AgNPs show $10 \%$ increase in the hydrodynamic diameter in the presence of HSA, while Cur-AgNPs show a $30 \%$ increase. This indicates that Cur-AgNPs have a greater degree of interactions with HSA over the other two NP types. Brewer et al. ${ }^{16}$ have noted that a decrease in the zeta potential indicates the binding of BSA to the surface of the NPs. In the current case, we observe that zeta potentials of all the three NP systems decrease on exposure to HSA, indicating that HSA binds to the surface of all three AgNPs forming a PC. The mechanism of these interactions is investigated further below.

Absorption Characteristics of AgNP Interactions with HSA. An increase in the absorption intensity of serum albumins with the increasing NP concentration has been attributed to a complex formation between these proteins and NPs and has been used as an indicator of the type of interactions between NPs and serum proteins. ${ }^{1,3,11}$ As such, the interaction of a fixed amount of HSA with increasing concentrations of different AgNPs was initially investigated by monitoring the absorption features of HSA at ca. $279 \mathrm{~nm}$. The results in Figure 2A-C show an increase in the intensity and blue shift in the HSA peak at $279 \mathrm{~nm}$ with the increasing AgNPs concentration beyond a certain minimal concentration threshold. This suggests the possibility of ground state complex formation between AgNPs and HSA because dynamic collisions only affect the excited states of the molecules and have no effect on the absorption spectra. ${ }^{15}$ Similar effects of NPs on HSA absorption have been previously observed. Overall, the increase in the intensity of these interactions is significantly greater with phytochemicalcoated AgNPs over Ct-AgNPs, and Cur-AgNPs show stronger interactions with HSA over EGCG-AgNPs. These observations, coupled with the DLS and zeta potential studies (Supporting Information Table S1), suggest that the phytochemical surface coating on AgNPs plays a significant role in influencing their interaction with serum proteins.

To further assess whether binding of HSA to the surface of AgNPs causes any NP aggregation, the SPR absorption features of AgNPs at c.a. $400 \mathrm{~nm}$ were also monitored in the presence of HSA (Figure 2D-F). The SPR peaks observed at $412 \mathrm{~nm}$ (Cur-AgNPs) and $406 \mathrm{~nm}$ (EGCG-AgNPs) marginally redshifted to 413 and $412 \mathrm{~nm}$, respectively, while the $416 \mathrm{~nm} \mathrm{Ct}-$ AgNPs peak showed no significant change. The red shifts in the SPR peaks of the NPs indicate an increase in the overall NP hydrodynamic size, which further supports the DLS and zeta potential measurements (Supporting Information Table S1); because of phytochemical binding, the hydrodynamic diameter of AgNPs increases without causing NP aggregation.

Fluorescence Characteristics of AgNPs Interaction with HSA. The Trp-214 residue in HSA is highly sensitive to the changes in its microenvironment, thereby providing the intrinsic fluorescence emission of Trp as a good indicator of HSA interactions with other species. ${ }^{31}$ Quenching or decrease in the fluorescence intensity may indicate a variety of interactions such as molecular rearrangement, collisional quenching, or ground state complex formation. ${ }^{3,7}$ HSA showed an emission band at $346 \mathrm{~nm}$ on excitation at $295 \mathrm{~nm}$, while AgNPs showed no fluorescence emission at this wavelength, 

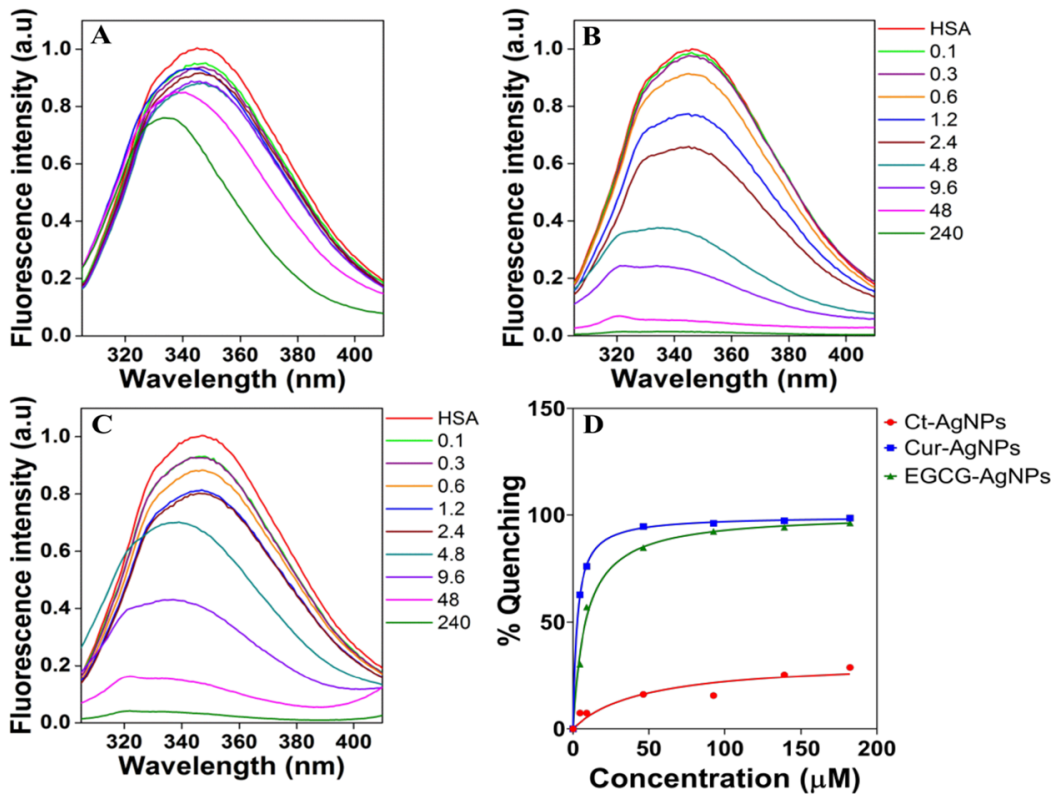

Figure 3. Fluorescence emission spectra in (A-C) depicting interactions of a fixed concentration of HSA $(3 \mu \mathrm{M})$ with an increasing concentration of AgNPs $(0-240 \mu \mathrm{M})$. HSA in the figure corresponds to the equivalent concentration of HSA (3 $\mu \mathrm{M})$ without nanoparticles. (D) Apparent dissociation constants as obtained using nonlinear regression fits in GraphPad Prism 7.02 (GraphPad, La Jolla, CA, USA). (A) Ct-AgNPs, (B) CurAgNPs, and (C) EGCG-AgNPs.

indicating the absence of overlapping fluorescence spectra from the AgNPs (Figure 3). The HSA concentration was maintained constant at $3 \mu \mathrm{M}$, whereas the AgNP concentration was varied between 0 and $240 \mu \mathrm{M}$. These concentrations were chosen to mimic a variety of potential NP exposure scenarios, such that either the protein concentration is up to 30 times greater than that of the AgNPs or the AgNP concentration is 80 times greater than the protein concentration.

As shown in Figure $3 \mathrm{~A}-\mathrm{C}$, in the presence of increasing concentrations of AgNPs, the Trp fluorescence was quenched in a NP concentration-dependent manner, with the largest degree of quenching caused by Cur-AgNPs, followed by EGCG-AgNPs and the least quenching observed with CtAgNPs. Because the efficiency of quenching depends on the proximity of the quencher to the fluorophore, ${ }^{17}$ these results indicate that the AgNPs (quencher in the current case) bind to the HSA molecule (fluorophore in the current case) at or near its Trp residue in the subdomain IIA. Another interesting observation is an increasing blue shift in the fluorescence spectra of HSA with the increasing AgNP concentration. This shift in the fluorescence emission indicates a concentrationdependent effect of AgNPs on HSA, such that its chromophore (Trp residue) has been brought into a more hydrophobic surrounding. 1,11,32 To validate the role of the hydrophobic environment on blue shifts of HSA fluorescence signatures, we performed additional experiments, wherein a fixed amount of HSA in the phosphate buffer was independently exposed to increasingly higher concentrations ( $\mathrm{v} / \mathrm{v}$ wrt water) of less polar solvents such as methanol and ethanol. It is clear from Figure S2 (Supporting Information) that the hydrophobic environment leads to the blue shift in the HSA fluorescence peaks. Another interesting observation was that HSA fluorescence increased in the presence of a nonpolar environment. This increase in fluorescence with the alcohols could be due to the change in the protein structure, exposing the tryptophan molecule; however, with the AgNPs, this was not observed because of interactions of the AgNPs at or near the tryptophan.
This supports our findings that the blue shift is due to the change in the hydrophobicity of the protein structure. Overall, these results signify that the AgNPs are capable of altering the structure of the HSA molecules.

From the fluorescence data, the dissociation constants $\left(K_{\mathrm{d}}\right)$ of HSA-AgNP interactions were calculated to determine the affinity of AgNPs with HSA (Figure 3D). ${ }^{26}$ It is clear that the affinity of HSA toward Cur-AgNPs is the highest with a $K_{\mathrm{d}}$ of $2.9 \mu \mathrm{M}$, followed by EGCG-AgNPs $(8.55 \mu \mathrm{M})$ and Ct-AgNPs $(28.99 \mu \mathrm{M})$. In particular, Cur-AgNPs and EGCG-AgNPs show 10 and 3 times higher affinity toward HSA over Ct-AgNPs. These high affinities for Cur-AgNPs and EGCG-AgNPs to HSA are similar to the affinity observed with polyethylene glycol and glucose coated FePt NPs to HSA, ${ }^{33-35}$ while the Ct-AgNPs are closer to the low affinity observed with polyacrylic acid-coated $\mathrm{CdSe} / \mathrm{ZnS} \mathrm{NPs}^{33,34}$ This supports that the nature of the capping agent over the NP surface plays a significant role in determining their interactions with serum proteins. Notably, while quenching of fluorescence emission during $\mathrm{NP}$-protein interactions has been previously observed, ${ }^{1-3,11,15,31}$ these studies have been restricted to studying a single type of NP, without considering the role of the surface corona.

It is acknowledged that free curcumin and EGCG molecules are known to show a phytochemical concentration-dependent increase in HSA fluorescence quenching. ${ }^{36,37}$ However, interactions of pristine phytochemicals with HSA lead to red shifts in the fluorescence emission, which is indicative of shifts toward a more polar environment. ${ }^{37}$ This is in contrast to phytochemical-coated AgNPs in the current case, where a blue shift (shifts toward a more nonpolar environment) in fluorescence emission is observed. Further, it was previously noted $^{38}$ that the hydroxyl groups on the galloyl moiety and on the B-ring of pristine EGCG are responsible for interactions with HSA. In the current case involving NPs, if the FTIR spectra of the Cur-AgNPs and EGCG-AgNPs are considered (Supporting Information Figure S3), a change in the phenolic groups of the phytochemicals on the surface of the AgNPs is 


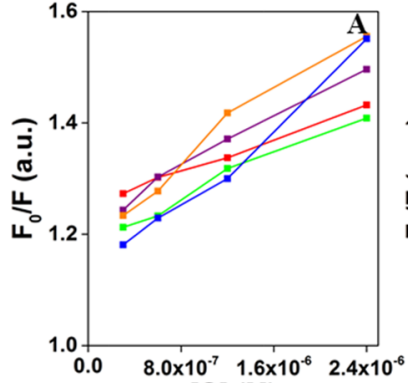

[Q] (M)

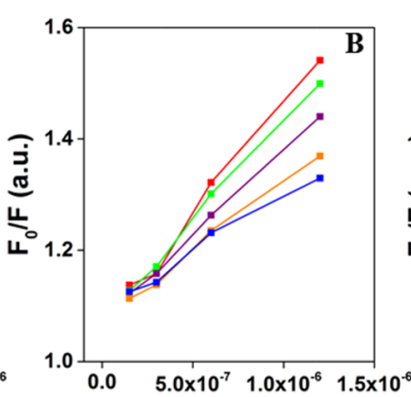

[Q] M

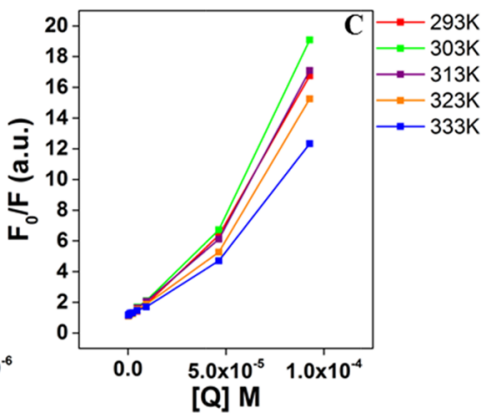

Figure 4. Stern-Volmer plots for (A) Ct-AgNPs, (B) Cur-AgNPs, and (C) EGCG-AgNPs at different temperatures.

Table 1. Stern-Volmer Constants and Thermodynamic Parameters for Cur-AgNPs

\begin{tabular}{|c|c|c|c|c|c|c|}
\hline temperature $(\mathrm{K})$ & $K_{\mathrm{SV}}\left(10^{4} \mathrm{M}^{-1}\right)$ & $k_{\mathrm{q}}\left(\times 10^{13} \mathrm{M}^{-1} \mathrm{~s}^{-1}\right)$ & $K\left(\times 10^{3} \mathrm{M}^{-1}\right)$ & $\Delta G^{\circ}\left(\mathrm{kJ} \mathrm{mol}{ }^{-1}\right)$ & $\Delta H^{\circ}\left(\mathrm{kJ} \mathrm{mol}^{-1}\right)$ & $\Delta S^{\circ}\left(\mathrm{J} \mathrm{mol}^{-1} \mathrm{~K}^{-1}\right)$ \\
\hline 293 & 33.49 & 3.35 & 4.80 & -54.02 & 81.75 & 184.63 \\
\hline 303 & 40.03 & 4.00 & 3.13 & -17.80 & 44.29 & 58.89 \\
\hline 313 & 35.91 & 3.59 & 1.38 & 17.22 & 9.22 & -54.99 \\
\hline 323 & 30.64 & 3.06 & 0.43 & 51.16 & -23.67 & -158.45 \\
\hline 333 & 24.92 & 2.49 & 0.11 & 84.11 & -54.59 & -252.74 \\
\hline
\end{tabular}

Table 2. Stern-Volmer Constants and Thermodynamic Parameters for EGCG-AgNPs

\begin{tabular}{|c|c|c|c|c|c|c|}
\hline temperature $(\mathrm{K})$ & $K_{\mathrm{SV}}\left(10^{4} \mathrm{M}^{-1}\right)$ & $k_{\mathrm{q}}\left(\times 10^{13} \mathrm{M}^{-1} \mathrm{~s}^{-1}\right)$ & $K\left(\times 10^{3} \mathrm{M}^{-1}\right)$ & $\Delta G^{\circ}\left(\mathrm{J} \mathrm{mol}^{-1}\right)$ & $\Delta H^{\circ}\left(\mathrm{kJ} \mathrm{mol}{ }^{-1}\right)$ & $\Delta S^{\circ}\left(\mathrm{J} \mathrm{K}^{-1} \mathrm{~mol}^{-1}\right)$ \\
\hline 293 & 15.04 & 1.50 & 7.53 & -4.25 & 5.02 & 14.53 \\
\hline 303 & 16.16 & 1.62 & 12.36 & -2.07 & 2.72 & 6.83 \\
\hline 313 & 18.42 & 1.84 & 14.27 & 0.05 & 0.57 & -0.15 \\
\hline 323 & 16.38 & 1.64 & 14.74 & 2.10 & -1.44 & -6.49 \\
\hline 333 & 14.39 & 1.44 & 6.62 & 4.08 & -3.34 & -12.27 \\
\hline
\end{tabular}

observed. This supports the fact that the chemical structures of phytochemicals change during reduction of $\mathrm{Ag}^{+}$ions and simultaneous surface capping of AgNPs. Therefore, it is not surprising that the interaction of phytochemical-coated AgNPs with HSA is not necessarily the same as the previously observed interaction of pristine phytochemicals with serum proteins. The authors recommend caution in making generalized predictions toward biological applicability of nanomaterials with a similar inorganic core but different surface cappings, or predicting the biological response of nanomaterials solely on the basis of the properties of pristine capping agents.

Fluorescence Quenching Mechanism. An interaction between a fluorophore and a quencher may undergo one of the two types of quenching mechanisms, viz., dynamic (or collisional) quenching and static quenching (or complex formation). Dynamic quenching occurs when a fluorophore in an excited state is deactivated when it remains in contact with the quencher, whereas static quenching occurs when the quencher and the fluorophore form a nonfluorescent ground state complex. ${ }^{3,26}$ These two types of quenching can be differentiated by studying the interactions as a function of temperature. In a dynamic quenching scenario, when temperature is increased, diffusion of molecules occurs at a faster rate, leading to the ongoing interactions between the quencher and the fluorophore. Conversely, in a static quenching scenario, the nonfluorescent ground state complex remains stable with the increasing temperature.

Fluorescence emission spectra of HSA with increasing concentrations of AgNPs were obtained at temperatures ranging from 20 to $60{ }^{\circ} \mathrm{C}(293-333 \mathrm{~K})$. To determine the mechanism of quenching, the data were analyzed for the quenching constant using the Stern-Volmer eq 1

$$
\frac{F_{0}}{F}=1+K_{\mathrm{SV}}[\mathrm{Q}]
$$

where $F_{0}$ and $F$ denote the fluorescence intensities in the absence and the presence of the quencher (AgNPs), respectively, $[\mathrm{Q}]$ is the concentration of AgNPs, and $K_{\mathrm{SV}}$ is the Stern-Volmer quenching constant. ${ }^{3}$

The Stern-Volmer quenching plots for the AgNPs are shown in Figure 4, and the corresponding Stern-Volmer quenching constants are displayed in Tables 1, 2, and S2 (Supporting Information) for Cur-AgNPs, EGCG-AgNPs, and Ct-AgNPs, respectively. The $K_{\mathrm{Sv}}$ values for Cur-AgNPs show a consistent decrease with the increasing temperature from 30 to $60{ }^{\circ} \mathrm{C}$ (Table 1). This indicates that the quenching mechanism is predominantly due to static binding of HSA to Cur-AgNPs because a decrease in $K_{\mathrm{SV}}$ with an increasing temperature indicates complex formation or static quenching. ${ }^{1,3,39}$ The $K_{\mathrm{SV}}$ values for EGCG-AgNPs, on the other hand, decrease only in the $40-60{ }^{\circ} \mathrm{C}$ range with the increasing temperature, whereas at a lower temperature range $\left(20-40{ }^{\circ} \mathrm{C}\right)$, an increase in $K_{\mathrm{SV}}$ is observed (Table 2). This suggests that at the physiological temperature of $37{ }^{\circ} \mathrm{C}$, EGCG-AgNPs exhibit dynamic quenching. However, as temperature increases, there is an evidence of static quenching. Notably, even in the case of CurAgNPs, an increase in $K_{\mathrm{SV}}$ indicative of dynamic quenching is observed at lower temperatures $\left(20-30{ }^{\circ} \mathrm{C}\right)$. The comparisons between two phytochemical-coated AgNPs at the physiological temperature suggests that Cur-AgNPs interact more strongly with HSA through a static complex formation, in contrast to EGCG-AgNPs, whose interactions with HSA are less stable because of dynamic interactions. In contrast to phytochemical- 
coated AgNPs, citrate-capped NPs show dynamic interactions with HSA across the wider temperature range of $20-60{ }^{\circ} \mathrm{C}$.

Further extension of the Stern-Volmer equation allows calculation of the biomolecular quenching rate constant $\left(k_{\mathrm{q}}\right)$ as shown in eq 2

$$
\frac{F_{0}}{F}=1+K_{\mathrm{SV}}[\mathrm{Q}]=1+k_{\mathrm{q}} \tau_{0}[\mathrm{Q}]
$$

where $K_{\mathrm{SV}}$ is the Stern-Volmer quenching constant, $k_{\mathrm{q}}$ is the biomolecular quenching rate constant, and $\tau_{0}$ is the average lifetime of the fluorophore without the quencher. $F_{0}$ and $F$ denote the fluorescence intensities in the absence and the presence of the quencher (AgNPs), respectively, whereas $[\mathrm{Q}]$ is the concentration of AgNPs.

The fluorescence lifetime of the HSA is $10^{-8} \mathrm{~s}\left(\tau_{0}\right)^{3}$, and $k_{\mathrm{q}}$ is calculated using the formula $K_{\mathrm{SV}} / \tau_{0}$. In the case of dynamic quenching, the maximum possible scatter quenching collision constant of various quenchers with the biopolymer is $2.0 \times 10^{10}$ $\mathrm{M}^{-1} \mathrm{~s}^{-13}$.,18 The $k_{\mathrm{q}}$ values obtained for all the three NPs are in the order of $10^{13} \mathrm{M}^{-1} \mathrm{~s}^{-1}$ (Tables 1 and 2 and Supporting Information Table S2), which are 3 orders of magnitude higher over the diffusion-controlled values encountered during dynamic interactions. This implies that at least to a finite extent, the AgNP-HSA complex is formed in the case of all NPs, leading to a degree of static interactions. ${ }^{11}$ The CurAgNPs show the highest $k_{\mathrm{q}}$ values, followed by EGCG-AgNPs and Ct-AgNPs, suggesting that the degree of static interactions between AgNPs and HSA is the largest in the case of CurAgNPs. Similarly, the $K_{\mathrm{SV}}$ values also indicated that the contribution of the static interactions was the largest in CurAgNPs $\left(30-60^{\circ} \mathrm{C}\right)$, followed by EGCG-AgNPs $\left(40-60^{\circ} \mathrm{C}\right)$, whereas $\mathrm{Ct}$-AgNPs did not show evidence of static interactions. These studies, in combination, suggest that phytochemicalcoated AgNPs show a complex interplay between dynamic and static interactions with HSA, such that interactions of HSA with Cur-AgNPs seem to be significantly stronger than those with EGCG-AgNPs.

Binding Constant and Thermodynamic Parameters. The double logarithmic regression curves of $\log \left[\left(F_{0}-F\right) / F\right]$ versus $\log [\mathrm{Q}]$ were plotted for the AgNPs, the intercept of which gives $K$, the binding constant (Supporting Information Figure S4). These binding constants are shown in Tables 1, 2, and S2 (Supporting Information) for Cur-AgNPs, EGCGAgNPs, and Ct-AgNPs, respectively. The $K$ value for Ct-AgNPs shows an increase with the temperature, indicating that the binding capacity increases as the temperature rises, and a similar observation has been made previously in the case of BSA interactions with citrate-capped AgNPs. ${ }^{3}$ The value of $K$ decreases with the increasing temperature for both the phytochemical-coated AgNPs, and this indicates a reduction in binding capacity with a concomitant rise in the temperature. Despite the $K_{d}$ values (Figure 3D) indicating high affinity of Cur-AgNPs and EGCG-AgNPs toward HSA, the decrease in the binding constant with temperature signifies the instability of the AgNPs-HSA complex in these cases. ${ }^{39,40}$

To determine the thermodynamic forces and the nature of binding of AgNPs to HSA, van't Hoff plots were used (Supporting Information, Figure S5). Because Ct-AgNPs exhibited a linear van't Hoff plot (Figure S5A), the changes in enthalpy and entropy were determined using the following equation

$$
\ln K=\frac{-\Delta H^{\circ}}{R T}+\frac{\Delta S^{\circ}}{R}
$$

where $K$ is the binding constant at the corresponding temperature $(T)$ in Kelvin; $R$ is the gas constant $\left(8.314 \mathrm{~J} \mathrm{~K}^{-1}\right.$ $\left.\mathrm{mol}^{-1}\right)$; and $\Delta H^{\circ}$ and $\Delta S^{\circ}$ correspond to the changes in enthalpy and entropy, respectively. ${ }^{3}$ However, because the trend for Cur-AgNPs and EGCG-AgNPs (Figure S5B,C) deviated from the linear dependence, polynomial equations were utilized as described previously. ${ }^{41}$ The dependence of $\ln K$ on $1 / T$ can be reduced to the quadratic form with three parameters $\left(\alpha_{0}, \alpha_{1}\right.$, and $\left.\alpha_{2}\right)$, which is represented in a polynomial equation as follows

$$
\ln K=\alpha_{0}+\frac{\alpha_{1}}{T}+\frac{\alpha_{2}}{T^{2}}
$$

Using these regression parameters, the thermodynamic parameters were calculated using the following equations ${ }^{41}$

$$
\begin{aligned}
& \Delta H^{\circ}=-R\left(\alpha_{1}+2 \frac{\alpha_{2}}{T}\right) \\
& \Delta S^{\circ}=R\left(\alpha_{0}-\frac{\alpha_{2}}{T^{2}}\right)
\end{aligned}
$$

and the Gibbs free energy $\left(\Delta G^{\circ}\right)$ was determined using the equation

$$
\Delta G^{\circ}=\Delta H^{\circ}-T \Delta S^{\circ}
$$

The nature of interactions between HSA and AgNPs can be determined from the changes in the enthalpy and entropy of the overall system. When $\Delta H^{\circ}$ and $\Delta S^{\circ}$ are both less than zero, such systems are dominated with van der Waals forces and hydrogen bonds; when $\Delta H^{\circ}$ and $\Delta S^{\circ}$ are both greater than 0 , this is characteristic of a system rich in hydrophobic forces; whereas when $\Delta H^{\circ}<0$ and $\Delta S^{\circ}>0$, this represents the system stabilized by electrostatic forces. ${ }^{3,18}$ Negative $\Delta G^{\circ}$ values indicate a spontaneous and thermodynamically favorable process. ${ }^{3,18}$ As such, the thermodynamic parameters obtained for Cur-AgNPs, EGCG-AgNPs, and Ct-AgNPs are displayed in Tables 1 and 2, and in Supporting Information Table S2, respectively. On the basis of these thermodynamic parameters, both the phytochemical-coated AgNPs exhibit hydrophobic interactions with HSA, and these interactions are thermodynamically favorable at ambient temperatures. However, with the increasing temperature, the interactions lose their spontaneity (positive $\Delta G^{\circ}$ ), and the van der Waals and hydrogen bonding start playing the major role in AgNP-HSA interactions. Pristine phytochemicals have also been previously observed to interact with HSA through van der Waals forces and hydrogen bonds, ${ }^{12,37}$ indicating that as the interaction temperature increases, the phytochemicals bound onto the NP surface obtain a greater degree of freedom to interact with the serum proteins in a manner that they interact in their free states. This also affirms that the surface coatings of NPs do play an important role in determining their interactions with the serum proteins. In contrast, Ct-AgNPs without any phytochemical coating (Supporting Information, Table S2) show a consistent behavior across a broader temperature range, which support a spontaneous thermodynamically favourable interaction with HSA molecules dominated by hydrophobic forces. This observation is in line with previous studies on citrate-capped NPs. ${ }^{3}$ Overall, a comparison between citrate- and phytochemicals-coated AgNPs suggests that while the latter do bind to 
HSA, the binding is less favorable than the interactions between Ct-AgNPs and HSA.

Effect of AgNPs on the Secondary Structure of HSA. Circular dichroism (CD) spectroscopy provides a convenient method for assessing the changes in the secondary structure, conformation, and stability of proteins in solution on interaction with NPs. Typically, the $\alpha$-helices of proteins exhibit two negative bands at 208 and $222 \mathrm{~nm}$, contributed by the $\pi-\pi^{*}$ and $n-\pi^{*}$ transitions of the peptide bonds of the $\alpha$ helix, respectively. ${ }^{1,40}$ The CD spectra of HSA in the presence and absence of AgNPs are shown in Figure 5. The $\alpha$-helix was

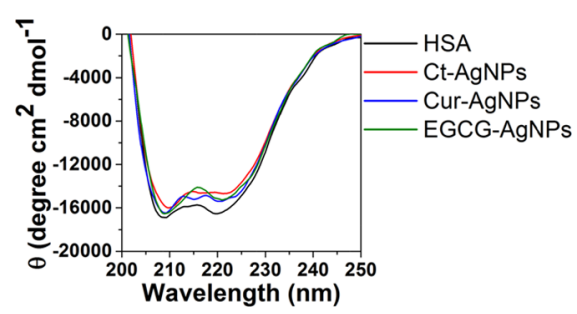

Figure 5. CD spectra of HSA in the absence and the presence of the AgNPs.

calculated from the mean residue ellipticity (MRE) values at $208 \mathrm{~nm}$ using the following equation

$$
\text { MRE }=\frac{\text { observed CD (mdeg) }}{C_{\mathrm{p}} n l \times 10}
$$

where $C_{\mathrm{p}}$ is the molar concentration of the protein, $n$ is the number of amino acid residues in HSA (585), and $l$ is the path length $(0.1 \mathrm{~cm}){ }^{40}$ The percentage of $\alpha$-helix content was calculated using the equation

$$
\alpha \text {-helix }(\%)=\frac{-\mathrm{MRE}_{208}-4000}{33000-4000} \times 10
$$

where $\mathrm{MRE}_{208}$ is the observed MRE at $208 \mathrm{~nm}, 4000$ is the MRE of the $\beta$-form and random coil conformation cross at 208 $\mathrm{nm}$, and 33000 is the MRE value of a pure $\alpha$-helix at $208 \mathrm{~nm}^{40}$

The $\alpha$-helix content, as obtained from CD spectroscopy of HSA in the phosphate buffer, showed a reduction from $44.4 \%$ in the case of free HSA to $38.6,41.8$, and $42.4 \%$ in the presence of Ct-AgNPs, Cur-AgNPs, and EGCG-AgNPs, respectively. This indicates that while all three AgNPs show a reduction in the $\alpha$-helices content of HSA, the reduction with Ct-AgNPs was the greatest. Between the two phytochemical-coated AgNPs, Cur-AgNPs showed greater reduction than EGCGAgNPs. The CD spectroscopy studies, therefore, support the fact that the interaction of phytochemical-coated AgNPs with human serum proteins causes fewer changes in the secondary structure of the protein, compared to citrate-stabilized AgNPs.

\section{CONCLUSIONS}

Citrate and sodium borohydride are the commonly employed reducing agents to prepare metal NPs of gold and silver. In comparison, while the use of phytochemicals to prepare metal NPs is not a common practice, the antioxidant capability of a large group of phytochemicals offers remarkable potential in their utility as "soft" reducing agents for metal NP synthesis. The inherent biocompatibility of a large number of these phytochemicals also has the potential to employ phytochemical-coated NPs for a myriad of biomedical applications. It is acknowledged that the interaction of sodium borohydride or citrate-capped metal NPs with serum proteins has been widely studied, and that most of these studies have focussed on BSA. ${ }^{1,3,16}$ There have been a selected few studies involving phytochemicals; however, these studies have employed the whole plant extracts for NP synthesis without using individual phytochemicals. ${ }^{11,15}$ These studies involving plant extracts for NP production do not provide molecular-level interaction insights and may lack the necessary aspects of quality control and precision manufacturing required for the intended biomedical use. Therefore, this study has, for the first time, obtained new insights into the human serum protein interactions of two phytochemical-stabilized metal NPs (CurAgNPs and EGCG-AgNPs) while comparing their performance with the widely reported Ct-AgNPs.

One of the key findings of our study is that the investigated phytochemical-capped AgNPs initially interact with HSA more strongly compared to Ct-AgNPs; however, the resultant NPHSA complexes subsequently become less stable in the case of the former, which causes a lesser degree of changes in the protein conformation during interactions. Another key difference observed between these two groups of AgNPs is that while under ambient conditions, all three types of AgNPs interacted with HSA predominantly via hydrophobic forces; however, with increasing temperatures (including at the physiological temperature), phytochemical-stabilized AgNPs showed a shift toward van der Waals and $\mathrm{H}$-bonding interactions. If compared within the phytochemical-capped group of AgNPs, while Cur-AgNPs showed static interactions with HSA, the EGCG-AgNPs interacted in a dynamic fashion. The outcomes of our study reveal that the use of phytochemicals as NP surface ligands offers new opportunities in controlling the interaction of NPs with complex biological fluids. For instance, differential interactions of different surface coatings of NPs with serum proteins have already been explored for developing "chemical nose-tongue" sensors. ${ }^{8}$ It is also noted that the formation of a PC once a NP comes in contact with biological fluids is a highly complex process. The current study focussed on understanding the interaction of phytochemical-coated NPs with HSA provides only a guiding tool, suggesting the potential benefits of phytochemicals in controlling the formation of a PC. However, further studies involving interactions of these NPs with complex fluids and full plasma will need to be performed to obtain a more comprehensive understanding of these interactions. Overall, the natural access to a repertoire of phytochemical molecules with diverse functional groups is likely to offer new opportunities in the field of nanodiagnostics and nanomedicine.

\section{MATERIALS \& METHODS}

Materials. Silver sulphate $\left(\mathrm{Ag}_{2} \mathrm{SO}_{4}\right)$, silver nitrate $\left(\mathrm{AgNO}_{3}\right)$ curcumin, EGCG, potassium hydroxide $(\mathrm{KOH}), \mathrm{HSA}$, monosodium phosphate $\left(\mathrm{NaH}_{2} \mathrm{PO}_{4}\right)$, disodium phosphate $\left(\mathrm{Na}_{2} \mathrm{HPO}_{4}\right)$, and dialysis membrane $(12 \mathrm{kDa}$ molecular weight cutoff) were purchased from Sigma-Aldrich (Castle Hill, NSW, Australia). A dialysis membrane was used after sensitizing by boiling in distilled water. All other materials were used as purchased without further modification. Sodium phosphate buffer ( $\mathrm{pH} 7.4$ ) was prepared by mixing $40.5 \mathrm{~mL}$ of $0.2 \mathrm{M}$ $\mathrm{Na}_{2} \mathrm{HPO}_{4}$ with $9.5 \mathrm{~mL}$ of $0.2 \mathrm{M} \mathrm{NaH}_{2} \mathrm{PO}_{4}$ and made up to a final volume of $100 \mathrm{~mL}$. Deionized Milli-Q water was used for all experiments.

AgNP Synthesis. The citrate-capped AgNPs (Ct-AgNPs) were synthesized using the citrate reduction method. Silver 
nitrate $(100 \mathrm{~mL}, 10 \mathrm{mM})$ was heated on a magnetic stirrer to $95^{\circ} \mathrm{C}$, and $2 \mathrm{~mL}$ of $1 \%$ trisodium citrate solution was added. The method of synthesis of curcumin-reduced AgNPs (CurAgNPs) and EGCG-reduced AgNPs (EGCG-AgNPs) was adapted from Selvakannan et al. ${ }^{23}$ To synthesize Cur-AgNPs, curcumin was dissolved in $0.1 \mathrm{M} \mathrm{KOH}$ and added to boiling water with equimolar aqueous silver sulphate solution. EGCGAgNPs were similarly prepared by adding equimolar concentrations of aqueous silver sulphate and EGCG solutions followed by $0.1 \mathrm{M} \mathrm{KOH}$. Heating was continued until the solutions turned yellowish brown, signifying the formation of AgNPs. The AgNPs were rotary-evaporated at $70 \mathrm{hPa}$, with the water bath heated to $70{ }^{\circ} \mathrm{C}$ and concentrated approximately to 10 times of the original concentration. The concentrated solutions were dialyzed for $24 \mathrm{~h}$ against deionized water at room temperature to remove excess $\mathrm{KOH}$, unreduced metal ions, and/or unbound molecules, if any.

Characterization of NPs. Synthesized AgNPs were characterized by UV-vis spectroscopy using a Varian Cary 50 spectrophotometer (Australia) from 200 to $800 \mathrm{~nm}$ in a quartz cuvette with a path length of $1 \mathrm{~cm}$. The nanoparticle core size was assessed by TEM. TEM images were obtained by dropcasting the dialyzed samples on carbon-coated copper grids and imaged using the JEOL 1010 TEM (USA) instrument operated at $100 \mathrm{kV}$. The hydrodynamic diameter and zeta potential of NPs were measured using a Malvern Nano-Zetasizer (UK) at $25{ }^{\circ} \mathrm{C}$. The samples were loaded into a folded capillary cell for the zeta potential measurements. The Smoluchowski methodology for aqueous media was employed, and a maximum of 100 runs was performed. ${ }^{42}$ For DLS, the samples were loaded into a polystyrene cuvette of $1 \mathrm{~cm}$ path length. For HSA-AgNP interaction studies, the molar equivalent concentration of $\mathrm{Ag}$ in AgNPs was determined by atomic absorption spectroscopy using a Varian PerkinElmer atomic absorption spectrometer. Throughout this study, the indicated AgNP concentration corresponds to the molar equivalent of $\mathrm{Ag}^{+}$ions.

Interaction of AgNPs with HSA. To study the interaction of the phytochemical-coated AgNPs with HSA, concentrationdependent studies were performed by incubating varying concentrations of the AgNPs $(0.1-240 \mu \mathrm{M})$ with $3 \mu \mathrm{M}$ of HSA in sodium phosphate buffer for $2 \mathrm{~h}$ at $4{ }^{\circ} \mathrm{C}$. All solutions were brought to room temperature for $30 \mathrm{~min}$ before acquiring UV-visible absorbance and fluorescence spectra. Fluorescence measurements were performed on a Jobin Yvon HORIBA FluoroMax 4 spectrophotometer (Japan). Because tryptophan is excited at $295 \mathrm{~nm}$ and produces an emission peak between 300 and $500 \mathrm{~nm},{ }^{26}$ fluorescence spectra were recorded in the range of $300-500 \mathrm{~nm}$ by exciting the reaction mixture at 295 $\mathrm{nm}$ at room temperature.

Further, to determine the binding affinity of AgNPs to HSA using the fluorescence spectra, the dissociation constant $\left(K_{\mathrm{d}}\right)$ was determined using eq 10

$$
Y=\frac{B_{\max } X}{K_{\mathrm{d}}+X}
$$

where $B_{\max }$ refers to the binding maxima and $X$ and $Y$ refer to the corresponding values from the $X$ and $Y$ axes. ${ }^{26}$ This equation was fitted using GraphPad Prism 7.02, GraphPad, La Jolla, CA.

For temperature-dependent studies, fluorescence spectra were collected from 20 to $70{ }^{\circ} \mathrm{C}$ at intervals of $10{ }^{\circ} \mathrm{C}$. From these data, the binding mechanism was elucidated using Stern-
Volmer plots by plotting $F_{0} / F$ versus [Q] at different temperatures. $^{43}$ The binding constant and the number of binding sites were determined using eq 11

$$
\log \frac{F_{0}-F}{F}=\log K+n \log [\mathrm{Q}]
$$

where $F_{0}$ and $F$ denote the fluorescence intensities in the absence and the presence of the quencher (AgNPs), respectively. $[\mathrm{Q}]$ is the concentration of the AgNPs, $K$ is the binding constant, and $n$ is the number of binding sites. ${ }^{3}$ Because the response of AgNPs at higher concentrations deviated from linearity, only low concentrations of AgNPs were used for calculations.

CD Spectroscopy. The conformational changes to HSA on interactions with the AgNPs were studied using a Jasco J-815 spectropolarimeter (USA) at room temperature $\left(25^{\circ} \mathrm{C}\right)$. The path length of the quartz cell used was $0.1 \mathrm{~cm}$. The protein concentration was $3 \mu \mathrm{M}$, and the AgNP concentration was 240 $\mu \mathrm{M}$. The CD spectra were collected from 190 to $300 \mathrm{~nm}$ using a scan speed of $50 \mathrm{~nm} / \mathrm{min}$ under a constant nitrogen flow. Three scans were averaged to improve the signal to noise ratio. The ellipticity values are expressed in terms of mean residue molar ellipticity $(\theta)$ in degree $\mathrm{cm}^{2} \mathrm{dmol}^{-1}$. Appropriate baseline corrections using sodium phosphate buffer were made.

\section{ASSOCIATED CONTENT}

\section{Supporting Information}

The Supporting Information is available free of charge on the ACS Publications website at DOI: 10.1021/acsomega.7b01878.

UV-visible absorption spectra of trisodium citrate, curcumin, and EGCG; nanoparticles size and zeta potential measurements; Stern-Volmer constants and thermodynamic parameters for Ct-AgNPs; fluorescent spectra of HSA in the presence of methanol and ethanol; FTIR spectra of the Cur-AgNPs and EGCG-AgNPs; double logarithmic regression plots of $\log \left[\left(F_{0}-F\right) / F\right]$ versus $\log [\mathrm{Q}]$; and van't Hoff plots (PDF)

\section{AUTHOR INFORMATION}

\section{Corresponding Authors}

*E-mail: vipul.bansal@rmit.edu.au. Phone: +61 399252121. Fax: +61 399253747 (V.B.).

*E-mail: ravi.shukla@rmit.edu.au. Phone: +61 3992529070. Fax: +61 399253747 (R.S.).

ORCID 8

Vipul Bansal: 0000-0002-3354-4317

\section{Author Contributions}

The manuscript was written through contributions of all authors. All authors have given approval to the final version of the manuscript. A.N.A. performed the experiments; V.B. and R.S. designed the study; all authors jointly analyzed the data and wrote the manuscript.

\section{Notes}

The authors declare no competing financial interest.

\section{ACKNOWLEDGMENTS}

A.N.A. acknowledges the Australian Technology Network for a Ph.D. scholarship support. R.S. acknowledges the Maxwell Eagle endowment fund. T.K.S. thanks the Commonwealth of Australia for an Endeavour Research Award for spending a research period at RMIT University. The authors acknowledge 
the Australian Research Council for fellowship and funding support (LP130100437-V.B. and R.S.; FT140101285-V.B.; DP170103477-V.B.). V.B. acknowledges the generous support of Ian Potter Foundation in establishing Sir Ian Potter NanoBioSensing Facility at RMIT University. Authors acknowledge the support from the RMIT Micro Nano Research Facility (MNRF) and RMIT Microscopy and Microanalysis Facility (RMMF) for technical assistance and providing access to characterization facilities.

\section{ABBREVIATIONS}

EGCG, epigallocatechin gallate; Cur, curcumin; HSA, human serum albumin; BSA, bovine serum albumin; AgNP, silver nanoparticle

\section{REFERENCES}

(1) Zhang, W.; Zhang, Q.; Wang, F.; Yuan, L.; Xu, Z.; Jiang, F.; Liu, $\mathrm{Y}$. Comparison of interactions between human serum albumin and silver nanoparticles of different sizes using spectroscopic methods. Luminescence 2015, 30, 397-404.

(2) Manivel, A.; Anandan, S. Spectral interaction between silica coated silver nanoparticles and serum albumins. Colloids Surf., A 2012, $395,38-45$.

(3) Mariam, J.; Dongre, P. M.; Kothari, D. C. Study of Interaction of Silver Nanoparticles with Bovine Serum Albumin Using Fluorescence Spectroscopy. J. Fluoresc. 2011, 21, 2193-2199.

(4) Lundqvist, M.; Stigler, J.; Elia, G.; Lynch, I.; Cedervall, T.; Dawson, K. A. Nanoparticle size and surface properties determine the protein corona with possible implications for biological impacts. Proc. Natl. Acad. Sci. U.S.A. 2008, 105, 14265-14270.

(5) Carnovale, C.; Bryant, G.; Shukla, R; Bansal, V. Size, shape and surface chemistry of nano-gold dictate its cellular interactions, uptake and toxicity. Prog. Mater. Sci. 2016, 83, 152-190.

(6) Boulos, S. P.; Davis, T. A.; Yang, J. A.; Lohse, S. E.; Alkilany, A. M.; Holland, L. A.; Murphy, C. J. Nanoparticle-protein interactions: a thermodynamic and kinetic study of the adsorption of bovine serum albumin to gold nanoparticle surfaces. Langmuir 2013, 29, 1498414996.

(7) Selva Sharma, A.; Ilanchelian, M. Comprehensive Multispectroscopic Analysis on the Interaction and Corona Formation of Human Serum Albumin with Gold/Silver Alloy Nanoparticles. J. Phys. Chem. B 2015, 119, 9461-9476.

(8) De, M.; Rana, S.; Akpinar, H.; Miranda, O. R.; Arvizo, R. R.; Bunz, U. H. F.; Rotello, V. M. Sensing of proteins in human serum using conjugates of nanoparticles and green fluorescent protein. Nat. Chem. 2009, 1, 461-465.

(9) Shaw, A. K.; Pal, S. K. Spectroscopic studies on the effect of temperature on $\mathrm{pH}$-induced folded states of human serum albumin. $J$. Photochem. Photobiol., B 2008, 90, 69-77.

(10) Canoa, P.; Simón-Vázquez, R.; Popplewell, J.; GonzálezFernández, A. A quantitative binding study of fibrinogen and human serum albumin to metal oxide nanoparticles by surface plasmon resonance. Biosens. Bioelectron. 2015, 74, 376-383.

(11) Ambika, S.; Sundrarajan, M. Green biosynthesis of $\mathrm{ZnO}$ nanoparticles using Vitex negundo L. extract: Spectroscopic investigation of interaction between $\mathrm{ZnO}$ nanoparticles and human serum albumin. J. Photochem. Photobiol., B 2015, 149, 143-148.

(12) Patra, D.; Barakat, C.; Tafech, R. M. Study on effect of lipophilic curcumin on sub-domain IIA site of human serum albumin during unfolded and refolded states: A synchronous fluorescence spectroscopic study. Colloids Surf., B 2012, 94, 354-361.

(13) Carril, M.; Padro, D.; del Pino, P.; Carrillo-Carrion, C.; Gallego, M.; Parak, W. J. In situ detection of the protein corona in complex environments. Nat. Commun. 2017, 8, 1542.

(14) Lo Giudice, M. C.; Herda, L. M.; Polo, E.; Dawson, K. A. In situ characterization of nanoparticle biomolecular interactions in complex biological media by flow cytometry. Nat. Commun. 2016, 7, 13475.
(15) Yue, H.-L.; Hu, Y.-J.; Chen, J.; Bai, A.-M.; Ouyang, Y. Green synthesis and physical characterization of $\mathrm{Au}$ nanoparticles and their interaction with bovine serum albumin. Colloids Surf., B 2014, 122, $107-114$.

(16) Brewer, S. H.; Glomm, W. R.; Johnson, M. C.; Knag, M. K.; Franzen, S. Probing BSA binding to citrate-coated gold nanoparticles and surfaces. Langmuir 2005, 21, 9303-9307.

(17) Mandal, S.; Hossain, M.; Devi, P. S.; Kumar, G. S.; Chaudhuri, $\mathrm{K}$. Interaction of carbon nanoparticles to serum albumin: elucidation of the extent of perturbation of serum albumin conformations and thermodynamical parameters. J. Hazard. Mater. 2013, 248-249, 238245.

(18) Markarian, S. A.; Aznauryan, M. G. Study on the interaction between isoniazid and bovine serum albumin by fluorescence spectroscopy: the effect of dimethylsulfoxide. Mol. Biol. Rep. 2012, 39, 7559-7567.

(19) Gelamo, E. L.; Tabak, M. Spectroscopic studies on the interaction of bovine (BSA) and human (HSA) serum albumins with ionic surfactants. Spectrochim. Acta, Part A 2000, 56, 2255-2271.

(20) Singh, D. V.; Bharti, S. K.; Agarwal, S.; Roy, R.; Misra, K. Study of interaction of human serum albumin with curcumin by NMR and docking. J. Mol. Model. 2014, 20, 2365.

(21) Li, M.; Hagerman, A. E. Role of the flavan-3-ol and galloyl moieties in the interaction of (-)-epigallocatechin gallate with serum albumin. J. Agric. Food Chem. 2014, 62, 3768-3775.

(22) Liu, S.; Guo, C.; Guo, Y.; Yu, H.; Greenaway, F.; Sun, M. Z. Comparative binding affinities of flavonoid phytochemicals with bovine serum albumin. Iran. J. Pharm. Res. 2014, 13, 1019-1028.

(23) Selvakannan, P. R.; Swami, A.; Srisathiyanarayanan, D.; Shirude, P. S.; Pasricha, R.; Mandale, A. B.; Sastry, M. Synthesis of aqueous Au core-Ag shell nanoparticles using tyrosine as a $\mathrm{pH}$-dependent reducing agent and assembling phase-transferred silver nanoparticles at the airwater interface. Langmuir 2004, 20, 7825-7836.

(24) Shukla, R.; Chanda, N.; Zambre, A.; Upendran, A.; Katti, K.; Kulkarni, R. R.; Nune, S. K.; Casteel, S. W.; Smith, C. J.; Vimal, J.; Boote, E.; Robertson, J. D.; Kan, P.; Engelbrecht, H.; Watkinson, L. D.; Carmack, T. L.; Lever, J. R.; Cutler, C. S.; Caldwell, C.; Kannan, R.; Katti, K. V. Laminin receptor specific therapeutic gold nanoparticles (198AuNP-EGCg) show efficacy in treating prostate cancer. Proc. Natl. Acad. Sci. U.S.A. 2012, 109, 12426-12431.

(25) Yallapu, M. M.; Nagesh, P. K. B.; Jaggi, M.; Chauhan, S. C. Therapeutic Applications of Curcumin Nanoformulations. AAPS J. 2015, 17, 1341-1356.

(26) Sharma, T. K.; Sapra, M.; Chopra, A.; Sharma, R.; Patil, S. D.; Malik, R. K.; Pathania, R.; Navani, N. K. Interaction of BacteriocinCapped Silver Nanoparticles with Food Pathogens and Their Antibacterial Effect. Int. J. Green Nanotechnol. 2012, 4, 93-110.

(27) Rodrigues, M. A.; Fernandes, J. N.; Ruggiero, R.; Guerra, W. Palladium Complex Containing Curcumin as Ligand: Thermal and Spectral Characterization. Am. J. Chem. 2012, 2, 157-159.

(28) Snitsarev, V.; Young, M. N.; Miller, R. M. S.; Rotella, D. P. The Spectral Properties of (-)-Epigallocatechin 3-O-Gallate (EGCG) Fluorescence in Different Solvents: Dependence on Solvent Polarity. PLoS One 2013, 8, No. e79834.

(29) Krukowski, S.; Karasiewicz, M.; Kolodziejski, W. Convenient UV-spectrophotometric determination of citrates in aqueous solutions with applications in the pharmaceutical analysis of oral electrolyte formulations. J. Food Drug Anal. 2017, 25, 717-722.

(30) Salatin, S.; Maleki Dizaj, S.; Yari Khosroushahi, A. Effect of the surface modification, size, and shape on cellular uptake of nanoparticles. Cell Biol. Int. 2015, 39, 881-890.

(31) Huang, S.; Qiu, H.; Lu, S.; Zhu, F.; Xiao, Q. Study on the molecular interaction of graphene quantum dots with human serum albumin: combined spectroscopic and electrochemical approaches. $J$. Hazard. Mater. 2015, 285, 18-26.

(32) Kim, C.; Savizky, R. M. A fluorescence quenching study of the human serum albumin-quercetin complex by addition of Copper(II), Nickel(II), and Manganese(II). Res. J. Pharm., Biol. Chem. Sci. 2013, 4, $765-795$. 
(33) Röcker, C.; Pötzl, M.; Zhang, F.; Parak, W. J.; Nienhaus, G. U. A quantitative fluorescence study of protein monolayer formation on colloidal nanoparticles. Nat. Nanotechnol. 2009, 4, 577.

(34) del Pino, P.; Pelaz, B.; Zhang, Q.; Maffre, P.; Nienhaus, G. U.; Parak, W. J. Protein corona formation around nanoparticles-from the past to the future. Mater. Horiz. 2014, 1, 301-313.

(35) Pelaz, B.; del Pino, P.; Maffre, P.; Hartmann, R.; Gallego, M.; Rivera-Fernández, S.; de la Fuente, J. M.; Nienhaus, G. U.; Parak, W. J. Surface Functionalization of Nanoparticles with Polyethylene Glycol: Effects on Protein Adsorption and Cellular Uptake. ACS Nano 2015, 9, 6996-7008.

(36) Barik, A.; Mishra, B.; Kunwar, A.; Indira Priyadarsini, K. Interaction of curcumin with human serum albumin: Thermodynamic properties, fluorescence energy transfer and denaturation effects. Chem. Phys. Lett. 2007, 436, 239-243.

(37) Maiti, T. K.; Ghosh, K. S.; Dasgupta, S. Interaction of (-)-epigallocatechin-3-gallate with human serum albumin: fluorescence, fourier transform infrared, circular dichroism, and docking studies. Proteins: Struct., Funct., Bioinf. 2006, 64, 355-362.

(38) Ishii, T.; Ichikawa, T.; Minoda, K.; Kusaka, K.; Ito, S.; Suzuki, Y.; Akagawa, M.; Mochizuki, K.; Goda, T.; Nakayama, T. Human serum albumin as an antioxidant in the oxidation of (-)-epigallocatechin gallate: participation of reversible covalent binding for interaction and stabilization. Biosci., Biotechnol., Biochem. 2011, 75, 100-106.

(39) Ambika, S.; Sundrarajan, M. Green biosynthesis of $\mathrm{ZnO}$ nanoparticles using Vitex negundo L. extract: Spectroscopic investigation of interaction between $\mathrm{ZnO}$ nanoparticles and human serum albumin. J. Photochem. Photobiol., B 2015, 149, 143-148.

(40) Yue, H.-L.; Hu, Y.-J.; Chen, J.; Bai, A.-M.; Ouyang, Y. Green synthesis and physical characterization of Au nanoparticles and their interaction with bovine serum albumin. Colloids Surf., B 2014, 122, $107-114$.

(41) Galaon, T.; David, V. Deviation from van't Hoff dependence in RP-LC induced by tautomeric interconversion observed for four compounds. J. Sep. Sci. 2011, 34, 1423-1428.

(42) Munusamy, P.; Wang, C.; Engelhard, M. H.; Baer, D. R.; Smith, J. N.; Liu, C.; Kodali, V.; Thrall, B. D.; Chen, S.; Porter, A. E.; Ryan, M. P. Comparison of $20 \mathrm{~nm}$ silver nanoparticles synthesized with and without a gold core: Structure, dissolution in cell culture media, and biological impact on macrophages. Biointerphases 2015, 10, 031003.

(43) Quenching of Fluorescence. In Principles of Fluorescence Spectroscopy; Lakowicz, J., Ed.; Springer US, 2006; pp 277-330. 